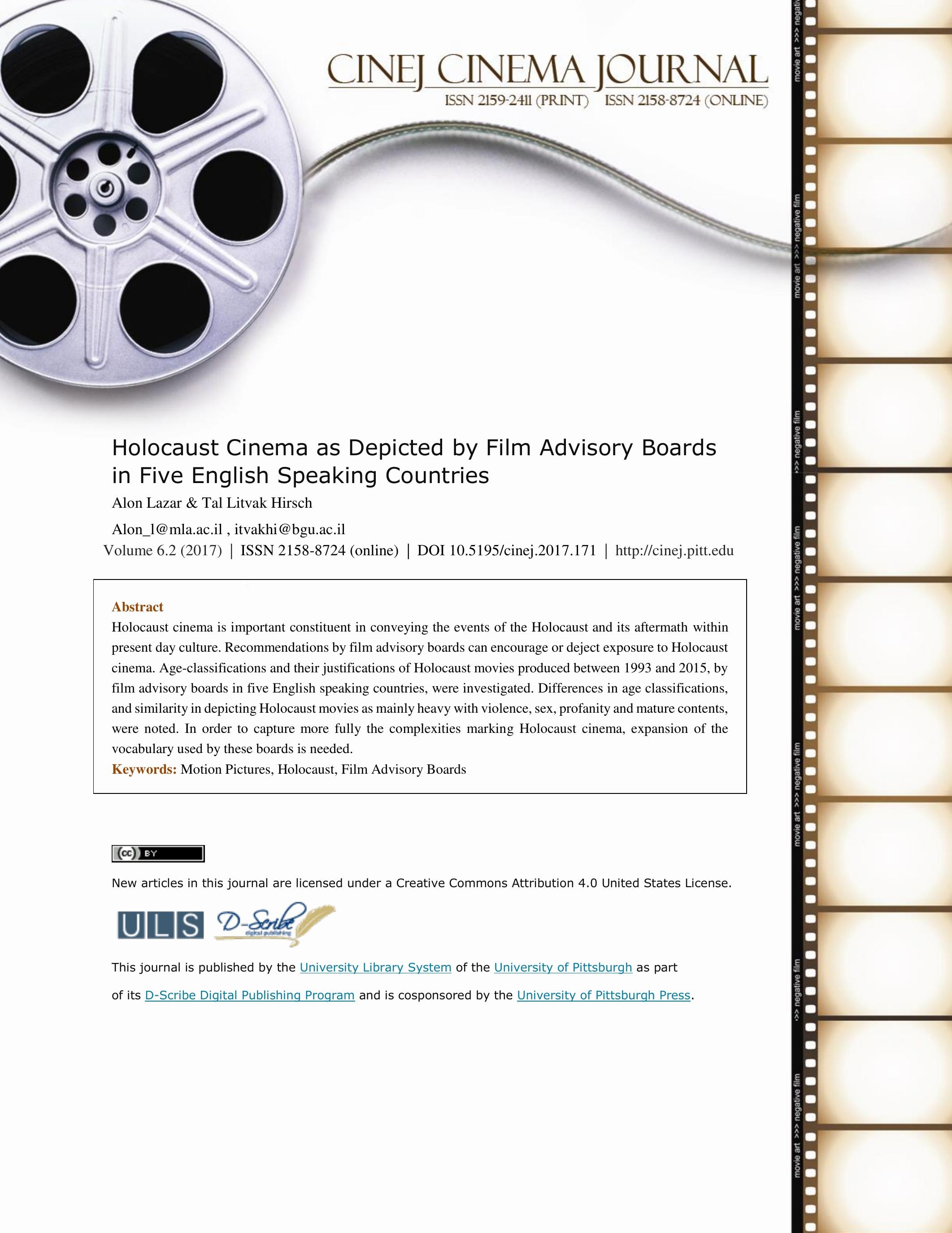




\section{Holocaust Cinema as Depicted by Film Advisory Boards in Five English Speaking Countries}

\section{Alon Lazar \& Tal Litvak Hirsch}

\section{Introduction}

The recent two decade has seen a growing interest in Holocaust cinema with the publications of dictionaries (Reimer \& Reimer, 2012), analysis of the nature of the movies considered Holocaust movies (Baron, 2005; Hirsch, 2004; Kerner, 2011; Saxton, 2008), as well as discussing the inclusion of Holocaust movies as part of Holocaust curricula (Gray, 2014; Metzger, 2012; Schweber, 2006).

The current work aims to add to these discussions by addressing a lacuna in research, namely, identifying the age-categories assigned to Holocaust movies produced between the years 1993 and 2015 and the justifications for these classifications, by movie advisory boards in five English speaking countries. Such an inspection would serve as an initial step in finding out how these social agencies treat Holocaust cinema, as through their age-categorizations and the justifications of them, they hold the power to bring closer or deter interested viewers to the memory of the Holocaust, as it is conveyed through this media.

Thus, the first section of the literature review deal with the nature of Holocaust cinema. The second section focuses on the inclusion of this genre of films within Holocaust curricula, and finally, the working of film advisory boards are discussed. 


\section{Holocaust cinema}

Baer (2001) identifies a deep and ongoing rift between those who reject the possibility that the culture industry in the form of television, new media, and films, can adequately represent the events of the Holocaust, and those who take a more positive stand on the matter.

Filmmakers crafting Holocaust movies are noted to diverge in the ways to aim to deal with the issues raised by its memory. Some present narratives, fictional or based upon actual events, depicting the actions of individuals and groups, be them victims, perpetrators, bystanders, resistors and helpers, prior to the Holocaust, during the years of annihilation, and how those who survived or were born after the end of World War Two, such as the descents of survivors or perpetrators as well as white supremacists and neo-Nazis, face the memory of the Holocaust (Baron, 2002; Baron, 2010; Owen, 2011; Reimer \& Reimer, 2012). Such productions, according to Hirsch (2004), are marked by the paradox of trying to visualize and narrate a trauma that cannot be captured in an image, namely events which are unrepresentable. As such, these movies, constitute a unique category of films, indentified by Hirsch as "posttraumatic cinema", one which aims to bring the viewer closer to a traumatic historical past, and as result might lead him/her to experience deep shock, numbness, and sense they have been forever changed, as they view extreme events which are radically out of their modes of interpreting and representing the world. Other filmmakers chose to discuss the memory of the Holocaust within genres such as comedy, science fiction (Baron, 2003), horror and sex-exploitation movies (Rapaport, 2003), or present alternative histories of it, most notably as is the case with Quentin Tarantino's Inglourious Basterds (Owen, 2011).

Eroticism, pornography, and violent sex scenes are frequent in many Holocaust movies, those produced in Europe and in other continents (Rapaport, 2003). When it comes to productions of Holocaust movies by American filmmakers, these are to large degree are influenced by 
conventions adopted from the genre of horror movies, a trend highly criticized by scholars (Picart \& Frank, 2006). Regardless of the mode of treatment, some of Holocaust movies reach global audience and highly praised critically (Baron, 2010).

\section{Holocaust movies within the curricula}

The Holocaust became in the western world a symbol of suffering, and through this prism, the suffering of indigenous groups in the United States, Canada, Australia and New Zealand is publicly debated and thought within the curricula (MacDonald, 2008). In Britain, as well as in other countries, Holocausts education is considered central within multicultural and citizenship education (Nesfield, 2015).

One way of bringing students closer to the events of the Holocausts, adopted by many teachers in various counties, is the inclusion of Holocausts movies within their classes. This state of affairs is part of the growing reliance of teachers on historical movies as part of their classes and the Holocaust being "widely accepted as morally crucial and educative in and of itself" (Schweber, 2006, p. 50). In Britain, teachers surveyed pointed to the movie Schindler's List as a valuable source within Holocaust education, and many of them noted that they might also incorporate in their classes the films The Pianist and The Boy in the Striped Pyjamas (Gray, 2014).

The Motion Picture Association of America (MPAA), the American film advisory board, classified the movies Schindler's List and The Pianist within the restrictive age category of R. Yet, educators who teach their students the Holocaust incorporate these two movies within their classes, and show them to students aged 15-16 years (Metzger, 2012). In that respect a potential underlying tension seems to exist between American educators and the MPAA as for the suitability of the contents included in two of the most renowned Holocaust movies for students. 


\section{Film advisory boards}

Movie advisory boards were designed to serve as primary social policy agencies, advising movie watchers and especially parents of theatrically-released movies which might contain contents inappropriate for their children (Potts \& Belden, 2009; Price, Palsson, \& Gentile, 2014). Film advisory boards operate worldwide and assign movies they scrutinize age-appropriate categories which diverge considerably. Thus the MPAA uses five categories ( $\mathrm{G}$-all ages admitted; PG - parental guidance suggested, some material may not be suitable for children; PG-13 - parents strongly cautioned, some material may be inappropriate for children under $13 ; \mathrm{R}$ - restricted, under 17 requires accompanying parent or adult guardian; and NC-17 - no one 17 and under admitted). In New Zealand, the Office of Film and Literature Classification (n.d.) assigns seven restricted labels (R13, R15M, R16, R18, RP13, RP16, and R), with additional three unrestricted labels (G, PG and $\mathrm{M})$.

Studies have documented cross-national differences when it comes to the leniency of advisory boards with regard to movie ratings. Thus for example, less age restrictive classifications were found to be assigned in France, Spain and Italy in comparison to the U.S., the U.K. and Australia (Leenders \& Eliashberg, 2011). Price et al. (2014) studied movies assigned with the R category by the MPAA and its equivalent age-category in additional 25 countries. It was found that among English speaking countries the MPAA provides more restrictive age-categories on movies heavy with profanity, yet, is fairly lenient when it comes to those marked by violence. The MPPA and its equivalent advisory board in Asian countries are highly restrictive when it comes to movies marked by sexual contents in comparison to their equals in Scandinavia, while Scandinavian boards are especially strict when it comes to violent films. These findings led Price et al. (2014) to conclude that "there is no universal consensus about what types of material is 
appropriate for children" (p. 249). Yet, these studies had not assessed the justifications for the age assigned classifications by these boards.

Beginning in 1993, the MPAA added the PG-13 category and alongside provides short justifications for the reasons why some movies are classified as age-restricted (Potts \& Belden, 2009). Since then, other advisory boards began to provide similar justifications. Potts and Belden (2009) analyzed the justifications provided by the MPAA for restricted movies rated PG, PG-13, $\mathrm{R}$ or NC-17, and detected four features. First, these authors identified several categories, termed as 'Core content terms'. These categories refer to justifications noting that a movie is marked as heavy with violence, sexuality, nudity, language, substance use, adult/thematic elements (indicating contents dealing with serious subjects or mature discussions regarding illness, abortion, coming-of age and verbal abuse), as well as other categories. Second, frequently, MPAA's justifications include 'Quantitative modifiers' which demarcate the duration or frequency of the core content terms, noting for example "a brief scene of violence" or "throughout use of language". Third, core content terms are also often accompanied by 'Qualitative modifiers' describing the potential emotional and/or psychological impact of some movie contents, as is the case with "intense violence," "mild language" or "shocking". Forth, at times, these justifications include also 'Context modifiers' which denote a case in which a core content theme is accompanied by an adjectives referring to a specific narrative or dramatic context such as 'zombie horror' or 'adolescent sexuality'.

\section{The current study}

Rapaport (2003) has suggested that "Popular culture plays an important role in linking words and images that create and sustain certain collective memories. Thus it provides useful cultural artifacts for critical attention that reveal and react to the broader sensibilities of an entire 
culture" (p. 78). The complex nature of Holocaust cinema calls for inspection of the vocabulary used by advisory boards when presenting interested members of their societies Holocaust movies. This is since, some of these movies hold the potential of serving for educational proposes (Gray, 2014; Metzger, 2012; Schweber, 2006), and hold the potential of deeply changing the viewer' world view (Hirsch, 2004) on the one hand.

On the other, some movies recognized as Holocaust films are more oriented for entertainment purposes and are marked by a tendency to emphasis more sensational aspects such as horror, violence and sex (Picart \& Frank, 2006; Rapaport, 2003), or are of humoristic nature.

In that respect, the age-categories and their justifications provided by national movie boards serve as cultural artifacts which help to identify how Holocaust movies are interpreted at the local level, as well as internationally. The two studies presented here aim to discern the ways Holocaust movies are presented to the general public by advisory boards in the English speaking countries. In the first study, the Canadian case is explored. Among English speaking countries, Canada is the only country in which rather than one authority is responsible to rate movies, six bodies, five of them responsible to provide the English speaking provinces with evolutions of movies, and one in the French speaking province of Quebec, independently rate movies. Among the five English speaking provinces an identical age classification system is employed, while the one in Quebec employs a different age-level system.

The comparison of the rating of Holocaust movies by Canadian provinces, although unique, would serve to identify at the national level differences are noted among the social agencies responsible to evaluate films. In the second study, the age-categories and their age-justifications by film advisory boards in four countries, the US, the UK, Australia and New Zealand, are assessed. In each of these countries there is one advisory board which serves as the authority when 
it comes to advising the public, and especially parents, as for the appropriateness of contents included in films. The ratings of Holocaust movies by film advisory boards in the English speaking provinces in Canada, the US, the UK, Australia and New Zealand, were chosen for the following reasons. Within these provinces and countries the Holocaust has been connected to various societal issues within the political and educational realms (MacDonald, 2008; Nesfield, 2015). Second, the justifications provided by these advisory boards could be discerned according to their core content terms, quantitative, qualitative and context modifiers as identified by the analysis by Potts and Belden (2009) of the justifications by the MPAA. This enables to point a national mode of treatment of Holocaust movies, as well as a cross-national comparison. This is not the case with the Irish Film Classification Office (IFCO) which presents its classifications within a table noting on a four point scale ranging from 'none' to 'strong' on how heavy is a movie on violence, drugs, sex/nudity, and language, alongside core content terms, quantitative, qualitative and context modifiers. In addition, and more importantly, IFCO reviewed only 36 Holocaust movies.

\section{Method}

A list of Holocaust movies released between the years 1993 (the first year the advisory boards following the MPAA began providing justifications) and 2015 was complied. In the absence of one all encamping and updated Internet site which presents the entire body of Holocaust movies, the dataset was assembled based on several sources. First were included the movies reviewed by Baron (2005), Kerner (2011) and Reimer and Reimer (2012). Second, a search was made within the Internet Data Base (IMDB), the Yad Vashem Visual Center and the Amazon.com Internet sites for Holocaust feature movies released theatrically and/or as DVDS. Thus were excluded documentaries, Television movies and personal and or/family movies. 
The search of IMDB, the Yad Vashem Visual Center and Amazon.com included the terms Holocaust, Jewish/Jew/Jews, Nazi/s, neo-Nazi/s, anti-Nazi/s, German/Germans, World War Two, concentration camp/s, ghetto/s, resistance/resistors, and rescuers.

Three hundred and eleven films were identified, and each was searched within the Internet site of the five Canadian advisory boards and each of the advisory board in the US, the UK, Australia and New Zealand. Considering the differences in the number of Holocaust movies reviewed by the boards studied here, in order to provide a valid comparison, the findings reported relate to movies discussed at least by three boards in Canada and at least three of the four boards of the US, the UK, Australia and New Zealand. In total, these findings represent the bulk of Holocaust movies reviewed during the last two decades by the boards in question.

Study 1: A national comparison of ratings of Holocaust movies by movie advisories in the English speaking provinces in Canada

Since 2003, the five movie advisory boards in the English speaking provinces make use of a very similar age-classification system to rate movies. All boards use the General $(\mathrm{G})$; Parental Guidance (PG); 14A - anyone under 14 years of age must be accompanied by an adult; and Adult categories. In British Colombia the 18/18A is not included in the age classification system, while in the remaining provinces, the $18 \mathrm{~A}$ category notes that those under 18 years of age must be accompanied by an adult. Minimal differences are noted in the case of the Restricted (R) category. In British Colombia, the R category states that "Restricted - No one under the age of 18 may view under any circumstances", in Alberta "Restricted - admittance restricted to persons 18 years of age or over", while in Manitoba, Ontario and Nova Scotia "Restricted - viewing, renting or buying is restricted to persons 18 years of age or over" (Manitoba Classification Board, n.d.). As for the 
type of justifications provided by these boards, in case of four of them, these include the ageclassification category and several key words such as violence, nudity and mature theme. The Alberta Film Classification board provided similar justifications provided during most of its years of activity. In recent years, this board it began to makes available a much richer descriptions of movies. These includes alongside the rational for classification such as coarse language, a synopsis of the movie, and perhaps more noticeably, several thematic elements of the movie. For example, in the case of the film Woman in Gold the board noted "The bonds of family; Restitution for Nazi atrocities; and History and memory". While these thematic elements are worthy of investigation, their uniqueness within the Canadian context and the fact that currently only a limited number of Holocaust movies were discussed in this manner, exceed the scope of the current investigation.

\section{Results}

Of the 311 movies surveyed, 160 were not evaluated by any of the Canadian boards researched here, 37 by one board, 25 by two boards, 34 by three boards, 25 by four boards and 29 by all five boards. Thus the final analysis focuses upon on 88 movies discussed by at least three of the five boards. Manitoba Film Classification Board provided the largest number of evaluations to Holocaust movies, reaching a total of 132 movies rated between the years 1993 and 2015. Seventyfour of these movies were assigned the 14A category, 41 the PG category, 12 the 18A category and five the R category. Fourteen of these movies were assigned an age-category classification, yet, no justification was given for this decision.

The Ontario Film Review Board (OFRB) reviewed 94 of the movies surveyed here. Since 2003 the category Adult Accompaniment (Film restricted to persons 14 years of age or older or to persons younger than 14 years of age who are accompanied by an adult) which was used by the OFRB during the years 1986-2003, is currently identified as the 14A category. The OFRB assigned 
the majority of Holocaust movies it evaluated $(n=52)$ the age classification of 14 (36 movies since 2003 received the 14 A category, and 16 movies reviewed between the years 1993 and 2003, the equivalent AA category), 36 movies were grouped under the PG category, three within the 18A category, and additional three in the R category. No justifications for these classifications were noted in the case of eighteen of these movies. The Nova Scotia board reviewed 92 movies, with 55 of them qualifying the 14A category, 24 in the PG category, and 8 in the R category. Twentysix of these movies were assigned an age-category classification, yet, no justification was given for this decision. The Alberta Film Boards evaluated 59 Holocaust movies, with 27 of them included in the 14A category, 24 in the PG category, and 8 in the 18A category. No justifications for these classifications were noted in the case of six of these movies. The board of British Columbia assessed 50 Holocaust movies, with 22 included in the PG category, 18 in the 14A category, 6 in the 18A category and 4 in the G category, with only two movies lack justifications for their classification.

Table 1 presents the content terms across movies as discussed by five Canadian advisory boards.

\begin{tabular}{|r|c|c|c|c|c|}
\hline Core content term & Manitoba & Ontario & $\begin{array}{c}\text { Nova } \\
\text { Scotia }\end{array}$ & $\begin{array}{c}\text { Alberta } \\
\text { Columbia }\end{array}$ & - \\
\hline Not recommended for children & 28 & 12 & 4 & 11 & - \\
\hline Adult/mature/thematic themes & 24 & 29 & 14 & 16 & 33 \\
\hline Violence & 23 & 19 & 13 & 22 \\
\hline Language/coarse /offensive & 15 & 20 & 12 & 4 & 15 \\
\hline Nudity & 8 & 11 & 4 & 9 & 1 \\
\hline May offend someone & 4 & 10 & 3 & - & - \\
\hline
\end{tabular}




\begin{tabular}{|c|c|c|c|c|c|}
\hline Tobacco/substance use & 2 & 3 & - & 1 & \\
\hline Sexuality & - & 2 & 1 & 6 & 10 \\
\hline $\begin{array}{r}\text { Holocaust images, themes or } \\
\text { elements }\end{array}$ & - & - & - & 3 & 1 \\
\hline Suggestive scenes & - & - & - & 2 & 15 \\
\hline Torture & - & 1 & - & 1 & \\
\hline Racism & - & - & - & 1 & \\
\hline
\end{tabular}

Table 1: Distribution of core content terms across movies

Two core content terms dominant the justifications made by the Canadian boards investigated here, namely, violence, especially noted in the case of the board of British Colombia, and profanity. All five boards also discussed the issues of nudity and sexual content to lesser degree. The core content of adult themes was noted in the justifications of four of the boards, and especially by Ontario and Manitoba boards. This core theme was completely absent in the case of their equivalent in British Colombia. The remaining core content terms were noted in justifications provided by three or less of the boards. Most notably, the Manitoba board emphasized that many Holocaust movies are not recommended for children and rarely explained why.

The members of the boards in British Colombia warned that some of these movies contain highly sexualized and suggestive contents. In comparison, rarely any of these movies were identified as discussing the Holocaust.

Table 2 presents the distribution of quantitative, qualitative and context modifiers as provided by the board of each province across movies and core content terms.

\begin{tabular}{|l|l|l|l|c|c|}
\hline Core content term & Manitoba & Ontario & $\begin{array}{c}\text { Nova } \\
\text { Scotia }\end{array}$ & Alberta & $\begin{array}{c}\text { British } \\
\text { Columbia }\end{array}$ \\
\hline Quantitative modifiers & & & & & \\
\hline
\end{tabular}




\begin{tabular}{|c|c|c|c|c|c|}
\hline Some & - & - & - & 5 & - \\
\hline Brief & - & - & - & 2 & - \\
\hline Frequent/thorough & - & - & 2 & 5 & 2 \\
\hline Infrequent/ occasional & - & - & - & 4 & - \\
\hline Several & - & - & 1 & - & 7 \\
\hline Providing an exact number & - & - & - & - & 17 \\
\hline \multicolumn{6}{|l|}{ Qualitative modifiers } \\
\hline Disturbing content & 10 & 9 & 5 & 9 & - \\
\hline Brutal/graphic & 2 & 4 & 2 & 1 & - \\
\hline Scene/s & 2 & 1 & 4 & 2 & 6 \\
\hline Frightening & 2 & - & 1 & 1 & - \\
\hline Gory & - & 1 & 1 & 3 & 1 \\
\hline Mild & - & - & - & 2 & - \\
\hline Explicit/pervasive/crude & - & - & 1 & 2 & 2 \\
\hline Intense & - & - & - & 2 & - \\
\hline Historical accuracy & - & - & - & 3 & - \\
\hline Detailed & - & - & - & 1 & - \\
\hline \multicolumn{6}{|l|}{ Context modifiers } \\
\hline Sexual violence & 1 & - & - & - & 2 \\
\hline Holocaust violence & - & - & - & 1 & 2 \\
\hline Suicide & - & - & - & 1 & \\
\hline Sexual language & - & - & - & 2 & 1 \\
\hline Historical setting & - & - & - & 1 & - \\
\hline War violence & - & - & - & 4 & - \\
\hline
\end{tabular}

Overall, the justifications provided by the Canadian boards studied here seldom include quantitative, qualitative and context modifiers. Of these it is worthy to note that the board of British Columbia is the only one to provide an exact number of quantitative modifiers such as noting as in the case of the movie Son of Saul "Two instances of coarse language; four scenes of nudity". When it comes to qualitative modifiers, all boards, although only fairly occasionally, note that the 
movies include disturbing content, when presenting Holocaust cinema to interested readers of their outputs.

\section{Discussion}

Within the English speaking provinces of Canada considerable differences are noted as for the number of Holocaust movies reviewed by their respective film boards during the period in question. Thus, the Manitoba Film Classification Board evaluated more than twice as much movies in comparison to its equivalents in the provinces of Alberta and British Columbia. The age classifications of these movies tend to fall mainly within the fairly restricted $14 \mathrm{~A}$ category, to lesser degree within the less restrictive PG category, and rarely within the highly restrictive classifications of 18A and R. Thus, a large fairly large number of potential movie watchers are eligible according to these Canadian boards to watch Holocaust movies. When it comes to the justifications provided by these social agencies, several similarities and some differences were observed. A consensus was noted when it comes to warning potential viewers that Holocaust cinema is marked by violence and profanity, and to lesser degree, as laden with nudity, sexual contents, and adult themes or as not suitable to young children. On the other hand, three of the boards suggested that at least some of the Holocaust movies reviewed include highly sexualized and including suggestive contents.

Considering that only a handful of movies were identified as Holocaust movies, this suggests that the members of these boards relay on a familiar vocabulary which is used to assess movies of all genres, rather than attempting to provide a more nuanced evolution of them. 


\section{Study 2: Cross-national comparisons of evaluations of Holocaust movies by film advisory boards in English speaking countries}

\section{Core}

As it turned out, 138 of 310 movies included in the database were screened only in their own country and/or in film festivals, or rated by other national boards. Fifty-one movies were rated by one of the boards discussed here, 23 of them by two of them, 33 by three boards and 67 by all four. The Office of Film \& Literature Classification (OFLC), the New Zealand advisory board, was found to rate a total of 119 Holocaust movies between the years 1993-2015, followed by the Australian Classification Board (ABC) and the (BFC) which provided evaluations for and 109 of the films, respectively. The MPAA rated 83 of these movies.

\section{Results}

In the final analysis are included 100 Holocaust movies, the majority of them $(n=67)$ reviewed by all the four boards discussed here, with the remaining 33 films by three of them. The $\mathrm{ABC}$ rated 96 of the movies studied here, the $\mathrm{BFC}$ and its equivalent in New Zealand classifying 94 of them, and the MPAA discussed 85 of them.

The $\mathrm{ABC}$ found the majority of the Holocaust movies noted here $(\mathrm{n}=49)$ to fit the $\mathrm{M}$ age category. Additional twenty-two movies were included in the MA15+ category, six in the PG category, three in the M18+ category and one in the R category. The BFC considered 38 Holocaust movies discussed here as fitting the 15 age-category, twenty-six as fitting the 12 age category, six the PG category, five the 18 age category, and one within the M category. Seven movies were 
classified to various age categories with no supplement justifications, but rather it was noted that "all known versions of this work passed uncut". The OFLC found the bulk of Holocaust researched here $(n=49)$ to fit the M category, eighteen the R16 category, five the PG category, four within the R18 classification, two were rated R13, and one as fitting the R15 category. Four movies were classified in either of these categories with no accompanying justification. Looking into the classifications by the MPAA, the majority of Holocaust movies reviewed here $(n=43)$ were included in the R age-category, eighteen were classified as PG-13, one as PG and another one as NC-17.

Table 3 presents the distribution of core content terms for each county across movies.

\begin{tabular}{|c|c|c|c|c|}
\hline Core content term & Australia & UK & $\begin{array}{c}\text { New } \\
\text { Zealand }\end{array}$ & US \\
\hline Violence & 58 & 44 & 64 & 44 \\
\hline Adult/mature/thematic themes & 55 & 1 & 4 & 13 \\
\hline Language/coarse /offensive & 38 & 41 & 40 & 37 \\
\hline Sexual/sexuality/sexual references & 30 & 27 & 29 & 41 \\
\hline Nudity & 9 & 5 & - & 21 \\
\hline Holocaust images, themes or elements & 6 & 9 & - & 5 \\
\hline Content that may disturb & - & - & 21 & 1 \\
\hline Reference to drugs and/or alcohol & - & & - & 1 \\
\hline Smoking & - & & - & 1 \\
\hline Threat & - & 6 & - & - \\
\hline Horror/gore/cruelty & 2 & 5 & 4 & 1 \\
\hline Psychiatric disorder theme & - & 1 & & - \\
\hline Distress & 1 & 1 & - & - \\
\hline Injury & - & 3 & - & - \\
\hline Rape & - & - & - & 1 \\
\hline Menace & - & 1 & & - \\
\hline
\end{tabular}


Three core content terms stand out as troublesome in the eyes of members of the film advisory boards surveyed here when it comes to Holocaust movies. These members view a considerable number of Holocaust movies to be marked by violence, most notably by the boards and New Zealand and Australia, profanity to similar degree by all boards, and sexuality, most notably by the MPAA. Other core content terms were noted quit differently by these boards. The ABC emphasized adult themes, its equivalent in New Zealand was concerned with contents that may disturb, and for the MPAA, nudity is quit an issue.

Straight forward identification of these movies as including Holocaust themes was only marginally noted, and none when it comes to the board of New Zealand. The remaining core contents, such as smoking, drugs and horror, were rarely discussed.

Table 4 presents the distribution of quantitative, qualitative and context modifiers for each county across movies and core content terms.

\begin{tabular}{|l|c|c|c|c|}
\hline Quantitative modifiers & Australia & UK & $\begin{array}{c}\text { New } \\
\text { Zealand }\end{array}$ & US \\
\hline Some & 2 & 1 & - & 39 \\
\hline Brief & - & 3 & - & 19 \\
\hline Frequent & - & 1 & - & - \\
\hline Infrequent/ occasional & 8 & 7 & - & - \\
\hline One & - & 1 & - & - \\
\hline Qualitative modifiers & & & & \\
\hline Strong & 23 & 57 & - & 17 \\
\hline High level/impact & 4 & - & - & - \\
\hline Medium level & 21 & - & 3 & - \\
\hline Moderate & 15 & 30 & - & - \\
\hline Mild & 2 & 7 & - & 1 \\
\hline Low level & 26 & - & 12 & - \\
\hline
\end{tabular}




\begin{tabular}{|l|c|c|c|c|}
\hline $\begin{array}{l}\text { Disturbing/distressing/ shocking } \\
\text { images/scenes }\end{array}$ & 1 & 1 & - & 14 \\
\hline Scene/s & 19 & 3 & 17 & 7 \\
\hline Intense & - & 1 & - & 1 \\
\hline Bad & - & - & - & - \\
\hline Offensive & - & - & - & 1 \\
\hline Graphic & - & 1 & - & 8 \\
\hline Emotional & - & 2 & - & - \\
\hline Context modifiers & & & & \\
\hline War violence/themes & - & 1 & - & - \\
\hline Wartime horror & - & 1 & - & - \\
\hline Sexual thereat/violence & - & 5 & 1 & - \\
\hline Sexualized nudity & - & 4 & - & - \\
\hline Sexual humor & - & - & - & 1 \\
\hline Bloody violence & - & 6 & - & 1 \\
\hline Suicide scene/theme & - & 1 & - & - \\
\hline Scene of torture and hanging & - & - & 1 & 1 \\
\hline Drug taking scenes & - & 1 & - & - \\
\hline Surgical detail & - & - & - & 1 \\
\hline Horror violence & - & - & - \\
\hline Child abuse references & - & - & - & - \\
\hline Sci-fi action violence & - & - & - & - \\
\hline
\end{tabular}

When it comes to the reference to quantitative modifiers, this mode of discussion marks the justifications provided by the MPAA, most notably that of 'some' and 'brief, while the other boards rarely, if at all, make use of them.

When it comes to qualitative modifiers certain boards, rather than others, make frequent use of them. The BFC provides justifications in which content terms depicting Holocaust movies are accompanied more so than others by the qualitative modifiers of 'strong' or 'moderate', while in the case of the $\mathrm{ABC}$ the qualitative modifier of 'medium level' dominants. The MPAA is marked for its use of the qualitative modifiers of 'disturbing/distressing/ shocking images/scenes', nearly 
missing in the assessments made by other boards. Of the three categories of modifiers, the one least noted is that of context modifiers, with any dominant pattern.

\section{Discussion}

Similar to cross-national studies looking at the age-classifications of movies of various genres by movie advisory boards (Leenders \& Eliashberg, 2011; Price et al., 2014), differences were noted with regard to the leniency of the boards with regard the Holocaust movies studied here. At first glance, the MPAA is the most restrictive of the boards studied here in terms of ageclassification of Holocaust movies. Yet, a deeper inspection pinpoints the BFC as the board most cautions of Holocaust cinema. This is reflected not only a fairly age-restrictive category of 15 years of age used by this board to warn the British audience from Holocaust cinema, but through the heavy use of the qualitative modifiers of 'strong' and 'moderate' accompanying the core contents.

The Australian and the New Zealand boards are the most lenient when it comes to Holocaust movies, as the majority of them were classified within the M category.

In both these countries, according the Internet sites of their movie advisory boards (Australian Classification Board, n.d.; Office of Film \& Literature Classification, n.d.), the M category is an advisory category which cautions that watching the movie is appropriate those who are 15 years and older, yet, does not prohibit exposure to it to all interested viewers. Of the four boards, OFLC is the most lenient, as rarely Holocaust movies are included in any of the seven restricted age-categories, and qualitative and quantitative modifiers are seldom used. In combination, in Australia and New Zealand, the local boards provide a cultural approval to interested viewers to watch Holocaust movies and leave them with the decision whether to watch them or not. This opens the possibility for teachers in these countries to choose from a wider variety 
from this genre of movies in comparison to their peers in the US and the UK, and leave the decision to parents in Australia and New Zealand, whether to allow their children to watch them or not. It would be seem best to demonstrate these differences in local sensitivities by looking into the ratings assigned to the three movies which had the greatest impact on popular conscience with regard to the Holocaust worldwide, namely, Schindler's List, Life is Beautiful and The Pianist (Saxton, 2008). The BFC categorized Schindler's List and The Pianist within the 15 age category, the first for "strong violence, threat, and strong language", while no justification was given for the latter, but rather it was noted that "all known versions of this work passed uncut". Life is Beautiful was assigned to the PG category for "Holocaust theme and mild horror". For the ABC both Schindler's List and Life is Beautiful fitted the M category, the first for "medium level violence, coarse language and adult themes" and the second for "adult themes". The Pianist was classified within the highly restrictive age category of MA15+ for "adult themes and high level violence". The OFLC rated both Schindler's List and Life is Beautiful within the M category, the first for "violence and offensive language," while in the case of the second no justification was provided. The highly restrictive age-category of R15 was assigned to the movie The Pianist for "violence and content that may disturb". The MPAA assigned the highly restrictive R category both to Schindler's List and The Pianist for "language, some sexuality and actuality violence", and for "violence and brief strong language", respectively. Life is Beautiful was assigned to the PG-13 category for "Holocaust-related thematic elements"

\section{General discussion}

Holocaust cinema occupy an important place in preserving the memory of the Holocaust (Rothe, 2011), attract the attention of movie makers (Baer, 2001), and serve educators to bring 
closer their students to actions and fates of those who witnessed it firsthand (Gray, 2014; Metzger, 2012; Schweber, 2006).

The study looked at the age-categories assigned to Holocaust movies and the justifications given for these classifications in five countries, in which English is the predominate language. The findings first suggest, that during the period studied here, while considerable number of Holocaust movies were produced relatively a small number of them were appraised by the film movie boards in question, and thus, the audience in their respective countries is exposed to a limited number of them.

National differences, in the case of Canada, and cross-national differences, in the case of the UK, the US, Australia and New Zealand, were noted in the judgments of advisory boards movies with regard to the assigned age-categories of Holocaust movies and the justifications explaining them.

Which aspects of Holocaust cinema were noted by the boards, the more potentially sensational and/ or entertainment oriented movies such as comedies, science-fiction, horror and alternative history marked by violence and sex, or conversely the perspective changing (Hirsch, 2004) or the educational (Schweber, 2006)?

When members of the five English speaking societies studied here consult the recommendations made by film advisory boards, they find that the majority of Holocaust movies reviewed during the last two decades are depicted as heavy with violence and sex, two aspect noted to mark Holocaust cinema (Picart \& Frank, 2006; Rapaport, 2003), as well as profanity. These three contents mark film productions of the last two decades (Leone \& Houle, 2006), and Holocaust movies, as the current findings suggest, are not exempted from this trend. An alternative explanation is that the current vocabulary serving the members of these members when evaluating 
Holocaust movies is too narrow and masks the differences between the various strands within Holocaust cinema. This is most notable when it comes to the core content term of violence, emphasized by all of the boards. The violence depicted in Holocaust movies dealing with the years preceding World War Two and during the war, should be placed within the unique historical context of persecution and attempt of annihilation, a type of violence a world apart from the one marking crime and thriller movies and/or horror movies, and even movies dealing with presentday racism by white supremacists and new-Nazis. As it turned out rarely, violence is discussed within the context of war in the justifications studied here.

Furthermore, and perhaps more importantly, only very few movies were identified as dealing with the Holocaust. Rather, references were made to adult/mature contents, especially in the case of the boards of Ontario and Manitoba in Canada, as well as in Australian, to contents that may disturb, noted by its equivalent in New Zealand, and pointing that some movies are not recommended for children as was the case with the board of Manitoba. Yet, these core content terms seem too vague and unclear in the current context. It seems that when it comes to Holocaust cinema, film advisory boards need to provide a unique category of Holocaust cinema, one which includes a suited vocabulary of justifications which address it in its full complexities. While the section entitled "thematic elements" included in recent evaluations provided by the Alberta Film Board seems as an initial step in that direction, a more refined and genre-specific categorization, one which will also include the traumatic and educative aspects of Holocaust movies, seems necessary. Such justifications would serve both parents and educators when deciding which Holocaust movies are suitable to watch by those under their care.

The current exploratory research focused upon the working of movie advisory boards in English speaking countries which had not experienced on their soil the years of persecution and annihilation marking the Holocaust. Expanding research to study how Holocaust movies are 
regarded by film boards in countries which carry the burden of these years, as well as other countries outside of Europe which do not, is needed to pinpoint similarities and differences in the cultural reception of the genre of films by these social agencies.

\section{BIBLIOGRAPHY}

Baer, A. (2001). Consuming history and memory through mass media products. European Journal of Cultural Studies, 4(4), 491-501.

Baron, L. (2010). Holocaust and genocide cinema: Crossing disciplinary, genre, and geographical borders: Editor's introduction. Shofar: An Interdisciplinary Journal of Jewish Studies, 28(4), 1-9.

Baron, L. (2005). Projecting the Holocaust into the present: The changing focus of contemporary Holocaust cinema. Lanham: Rowman \& Littlefield Publishers.

Baron, L. (2003). X-Men as J Men: The Jewish subtext of a comic book movie. Shofar: An Interdisciplinary Journal of Jewish Studies, 22(1), 44-52.

Baron, L. (2002). Holocaust iconography in American feature films about Neo-Nazis.

Film \& History, 32(2), 38-47.

Gray, M. (2014). The Boy in the Striped Pyjamas: A blessing or curse for Holocaust education?. Holocaust Studies, 20(3), 109-136.

Kerner, A. (2011). Film and the Holocaust: New perspectives on dramas, documentaries, and experimental films. A\&C Black.

Hirsch, J. (2004). Afterimage: Film, trauma and the Holocaust. Philadelphia: Temple University Press.

Leone, R., \& Houle, N. (2006). 21st century ratings creep: PG-13 and R. Communication Research Reports, 23(1), 53-61.

MacDonald, D. B. (2008). Identity politics in the age of genocide: The Holocaust and historical representation. New York, NY: Routledge.

Metzger, S. A. (2012). The borders of historical empathy: Students encounter the Holocaust through film. The Journal of Social Studies Research, 36(4), 387-410.

Leenders, A.A. M., \& Eliashberg, J. (2011). The antecedents and consequences of restrictive agebased ratings in the global motion picture industry. International Journal of Research in Marketing, 28(4), 367-377.

Manitoba Classification Board (n.d.). Comparison of Canadian classification systems. http://www.gov.mb.ca/chc/mfcb/class3.html 
Nesfield, V. (2015). Keeping Holocaust education relevant in a changing landscape: seventy years on. Research in Education, 94(1), 44-54.

New Zealand's Office of Film \& Literature Classification. (n.d). http://www.classificationoffice.govt.nz/find-ratings/new-zealands-classification-labels.html.

Owen, A. S. (2011). Expertise, criticism and Holocaust memory in cinema. Social Epistemology, 25(3), 233-247.

Picart, C. J., \& Frank, D. A. (2006). Frames of evil: The Holocaust as horror in American film. Carbondale: Southern Illinois University Press.

Potts, R., \& Belden, A. (2009). Parental guidance: A content analysis of MPAA motion picture rating justifications 1993-2005. Current Psychology, 28(4), 266-283.

Price, J., Palsson, C., \& Gentile, D. (2014). What matters in movie ratings? Cross-country differences in how content influences mature movie ratings. Journal of Children and Media, 8(3), 240-252.

Rapaport, L. (2003). Holocaust pornography: Profaning the sacred in Ilsa, She-Wolf of the SS. Shofar: An Interdisciplinary Journal of Jewish Studies, 22(1), 53-79.

Reimer, R. C., \& Reimer, C. J. (2012). Historical dictionary of Holocaust cinema. Lanham, Maryland: Scarecrow Press.

Rothe, A. (2011). Popular trauma culture: Selling the pain of others in the mass media. New Brunswick, New Jersey: Rutgers University Press.

Saxton, L. (2008). Haunted images: Film, ethics, testimony and the Holocaust. New York, NY: Wallflower Press.

Schweber, S. (2006). "Holocaust fatigue" in teaching today. Social Education, 70(1), 48-55. 